TRANSACTIONS OF THE

AMERICAN MATHEMATICAL SOCIETY

Volume 339, Number 2, October 1993

\title{
INEQUALITIES FOR MIXED PROJECTION BODIES
}

\author{
ERWIN LUTWAK \\ Dedicated to Professor Helmut Groemer on the occasion of his 60th birthday
}

\begin{abstract}
Mixed projection bodies are related to ordinary projection bodies (zonoids) in the same way that mixed volumes are related to ordinary volume. Analogs of the classical inequalities from the Brunn-Minkowski Theory (such as the Minkowski, Brunn-Minkowski, and Aleksandrov-Fenchel inequalities) are developed for projection and mixed projection bodies.
\end{abstract}

Two decades ago, Bolker [5] observed that projection bodies (also known as zonoids) were objects of independent investigation in a number of mathematical disciplines such as measure theory, crystallography, optimal control theory, functional analysis, and geometric convexity. Since the appearance of Bolker's article, projection bodies have received considerable increased attention (see, for example, $[7,13,14,21,23,26,27,28,32,40,41]$ ). Also, new applications have appeared in combinatorics (see Stanley [36]), in stereology (see Betke-McMullen [4]), in stochastic geometry (see Schneider [32, 33]), in mathematical economics (see Hildenbrand [16]), and even in the study of random determinants (see Vitale [39]). A fascinating recent paper of Alexander [3] demonstrates a close relationship between the study of zonoids and work on Hilbert's Fourth Problem.

Mixed projection bodies are related to ordinary projection bodies in the same way that mixed volumes are related to ordinary volume. The definition and some elementary properties of mixed projection bodies can be found in the classic volume of Bonnesen-Fenchel [6]. Support functions of mixed projection bodies were investigated by Chakerian [9]. Stability questions for mixed projection bodies are treated by Goodey [11] and Goodey-Groemer [12]. In [18] and [19], inequalities for the polars of mixed projection bodies were obtained. This article treats the corresponding inequalities for the mixed projection bodies themselves. Analogs of the classical mixed volume inequalities (such as the Brunn-Minkowski, Minkowski, and Aleksandrov-Fenchel inequalities) will be established for mixed projection bodies.

Since interest in zonoids is not limited to one discipline, an attempt is made to make this article reasonably self-contained.

Background material and notation regarding mixed volumes and mixed surface area measures is given in $\S 0$. The classical mixed volume inequalities are

Received by the editors January 14, 1991 and, in revised form, August 20, 1991.

1991 Mathematics Subject Classification. Primary 52A40.

Key words and phrases. Zonoids, projection bodies, mixed volumes.

Research supported, in part, by NSF Grants DMS-8704474 and DMS-8902550. 
listed in $\S 1$. Nothing in either of these sections is original. The statements are given for quick later reference and comparison. The reader may wish to consult some of the following references regarding material contained in the first two sections: Bonnesen-Fenchel [6], Busemann [8]. Leichtweiß [17], and the original works of Aleksandrov [1,2] and Fenchel-Jessen [10]. The excellent surveys of McMullen-Schneider [24] and Schneider [31] are also recommended.

Definitions and basic properties of mixed projection bodies are given in $\S 2$. Some of the material here can be found in [6, p. 45] or [18], some is folklore, and a bit is new.

In $\S 3$, an identity involving the mixed volumes of mixed projection bodies is presented. This easily established result (and some of its consequences) will play a significant role in the proofs of the inequalities to be presented in later sections.

A Minkowski inequality for mixed projection bodies (with equality conditions) is presented in $\S 4$. A uniqueness theorem for convex bodies, which involves the Quermassintegrals of mixed projection bodies, is also given here.

A general Aleksandrov-Fenchel inequality for mixed projection bodies is proven in $\S 5$. This inequality, when restricted to special mixed projection bodies, is obtained with equality conditions.

A Brunn-Minkowski inequality for mixed projection bodies, with equality conditions, is established in Section 6. A generalization (without equality conditions) is also derived.

\section{NOTATION AND BACKGROUND MATERIAL}

Let $\mathscr{K}^{n}$ denote the set of convex figures (compact, convex subsets) of Euclidean $n$-space, $\mathbf{R}^{n}$. Let $\mathscr{K}_{0}^{n}$ denote the subset of $\mathscr{K}^{n}$ consisting of the convex bodies (convex figures with nonempty interiors) in $\mathbf{R}^{n}$.

Let $h_{K}=h(K, \cdot): S^{n-1} \rightarrow \mathbf{R}$, denote the support function (restricted to the unit sphere) of $K \in \mathscr{K}^{n}$; i.e., for $u \in S^{n-1}$,

$$
h_{K}(u)=\max \{u \cdot x: x \in K\} \text {, }
$$

where $u \cdot x$ denotes the usual inner product of $x$ and $u$ in $\mathbf{R}^{n}$. Let $\delta$ denote the Hausdorff metric on $\mathscr{K}^{n}$; i.e., for $K, L \in \mathscr{K}^{n}$,

$$
\delta(K, L)=\left|h_{K}-h_{L}\right|_{\infty},
$$

where $\mid \cdot l_{\infty}$ denotes the sup-norm on the space of continuous functions, $C\left(S^{n-1}\right)$.

For $K_{1}, \ldots, K_{r} \in \mathscr{K}^{n}$ and $\lambda_{1}, \ldots, \lambda_{r} \geq 0$, the Minkowski linear combination $\lambda_{1} K_{1}+\cdots+\lambda_{r} K_{r} \in \mathscr{K}^{n}$ is defined by

$$
\lambda_{1} K_{1}+\cdots+\lambda_{r} K_{r}=\left\{\lambda_{1} x_{1}+\cdots+\lambda_{r} x_{r} \in \mathscr{K}^{n}: x_{i} \in K_{i}\right\} .
$$

It is trivial to verify that

$$
h\left(\lambda_{1} K_{1}+\cdots+\lambda_{r} K_{r}, \cdot\right)=\lambda_{1} h\left(K_{1}, \cdot\right)+\cdots+\lambda_{r} h\left(K_{r}, \cdot\right) .
$$

Of fundamental importance is the fact that the volume, $V\left(\lambda_{1} K_{1}+\cdots+\lambda_{r} K_{r}\right)$, of a linear combination of bodies defined by (0.2), can be expressed as a symmetric homogeneous $n$th degree polynomial in the $\lambda_{i}$ :

$$
V\left(\lambda_{1} K_{1}+\cdots+\lambda_{r} K_{r}\right)=\sum \lambda_{i_{1}} \cdots \lambda_{i_{n}} V_{i_{1} \cdots i_{n}},
$$


where the sum is taken over all $n$-tuples $\left(i_{1}, \ldots, i_{n}\right)$ of positive integers not exceeding $r$. The coefficient $V_{i_{1} \cdots i_{n}}$ (which is required to be symmetric in its subscripts) depends only on the bodies $K_{i_{1}}, \ldots, K_{i_{n}}$, and is uniquely determined by (0.4); it is called the mixed volume of $K_{i_{1}}, \ldots, K_{i_{n}}$, and is written as $V\left(K_{i_{1}}, \ldots, K_{i_{n}}\right)$. If $K_{1}, \ldots, K_{s} \in \mathscr{K}^{n}$, and $d_{1}, \ldots, d_{s}$ are nonnegative integers whose sum is $n$, then $V\left(K_{1}, d_{1} ; \ldots ; K_{s}, d_{s}\right)$ will denote the mixed volume with $d_{i}$ copies of $K_{i}$. The $d_{i}$ equal to 1 will often be suppressed. If $K, L \in \mathscr{K}^{n}$, and $0 \leq i \leq n$, then $V(K, n-i ; L, i)$ will usually be written as $V_{i}(K, L)$. For $0 \leq i<n$, write $W_{i}(K, L)$ for the mixed volume $V(K, n-i-1 ; B, i ; L)$, where $B$ is the unit ball of $\mathbf{R}^{n}$. The mixed volume $W_{i}(K, K)$ is usually written as $W_{i}(K)$ and is called the $i$ th Quermassintegral (or $i$ th mean projection measure) of $K$. Thus, $W_{0}(K)$ is the ordinary volume of $K$, while $W_{1}(K)$ is the surface area of $K$ divided by $n$. If $K_{1}, \ldots, K_{s}, L_{1}, \ldots, L_{t} \in \mathscr{K}^{n}$, and $d_{1}, \ldots, d_{s}$ are nonnegative integers (whose sum is $n-t$ ), and $\mathbf{C}=\left(L_{1}, \ldots, L_{t}\right)$, then $V\left(K_{1}, d_{1} ; \ldots ; K_{s}, d_{s} ; \mathbf{C}\right.$ ) will denote the mixed volume with $d_{i}$ copies of $K_{i}$ and a single copy of each of the $L_{i}$; i.e.,

$$
V\left(K_{1}, d_{1} ; \ldots ; K_{s}, d_{s} ; \mathbf{C}\right)=V\left(K_{1}, d_{1} ; \ldots ; K_{s}, d_{s} ; L_{1} ; \ldots ; L_{t}\right) .
$$

The mixed volume $V(K, n-t ; \mathbf{C})=V\left(K, n-t ; L_{1}, \ldots, L_{t}\right)$ will often be written as $V_{t}(K, \mathrm{C})$.

For $K \in \mathscr{K}^{n}$, and $u \in S^{n-1}$, let $K^{u}$ denote the image of the orthogonal projection of $K$ onto $\xi_{u}$, the $(n-1)$-dimensional subspace of $\mathbf{R}^{n}$ that is orthogonal to $u$. If $K_{1}, \ldots, K_{n-1} \in \mathscr{K}^{n}$, then write $v\left(K_{1}^{u}, \ldots, K_{n-1}^{u}\right)$ for the mixed volume of the figures $K_{1}^{u}, \ldots, K_{n-1}^{u}$ in the space $\xi_{u}$. For $K, L \in$ $\mathscr{K}^{n}$, write $v_{i}\left(K^{u}, L^{u}\right)$ for the mixed volume $v\left(K^{u}, n-i-1 ; L^{u}, i\right)$. For $v_{i}\left(K^{u}, B^{u}\right)$, write $w_{i}\left(K^{u}\right)$, and for the $(n-1)$-dimensional volume of $K^{u}$, write $v\left(K^{u}\right)$ rather than $w_{0}\left(K^{u}\right)$. It is well known, and easily shown, that for $K_{1}, \ldots, K_{n-1} \in \mathscr{K}^{n}$, and $u \in S^{n-1}$,

$$
v\left(K_{1}^{u}, \ldots, K_{n-1}^{u}\right)=n V\left(K_{1}, \ldots, K_{n-1}, \bar{u}\right),
$$

where $\bar{u}$ denotes the line segment joining $u / 2$ and $-u / 2$.

Associated with $K_{1}, \ldots, K_{n-1} \in \mathscr{K}_{0}^{n}$ is a Borel measure, $S\left(K_{1}, \ldots, K_{n-1} ; \cdot\right)$, on $S^{n-1}$, called the mixed surface area measure of $K_{1}, \ldots, K_{n-1}$, which has the property that for each $K \in \mathscr{K}^{n}$,

$$
V\left(K_{1}, \ldots, K_{n-1}, K\right)=\frac{1}{n} \int_{S^{n-1}} h_{K}(u) d S\left(K_{1}, \ldots, K_{n-1} ; u\right) .
$$

In fact, the measure $S\left(K_{1}, \ldots, K_{n-1} ; \cdot\right)$, can be defined by the property that (0.6) holds for all $K \in \mathscr{K}^{n}$.

\section{Mixed volumes}

One may view the mixed volume functional

$$
V: \underbrace{\mathscr{R}^{n} \times \cdots \times \mathscr{K}^{n}}_{n} \longrightarrow[0, \infty)
$$

as a polylinearization induced by the ordinary volume functional,

$$
V: \mathscr{K}^{n} \rightarrow[0, \infty),
$$


when $\mathscr{K}^{n}$ is endowed with the Minkowski linear structure (viz. (0.2)). The following is a list of the basic properties of the mixed volume functional:

$1_{v}$. It is symmetric and multilinear with respect to Minkowski linear combinations.

2 . Its diagonal form reduces to ordinary volume; i.e., for $K \in \mathscr{K}^{n}$,

$$
V(K, \ldots, K)=V(K) \text {. }
$$

$3 \mathrm{v}$. It is continuous with respect to the Hausdorff metric.

$4 \mathrm{v}$. It is invariant under independent translations of its arguments, and it is invariant under simultaneous unimodular linear transformations; i.e., if $K_{1}, \ldots, K_{n} \in \mathscr{K}^{n}$, and $\phi \in S L(n)$, then

$$
V\left(\phi K_{1}, \ldots, \phi K_{n}\right)=V\left(K_{1}, \ldots, K_{n}\right) .
$$

$5_{\mathrm{v}}$. It is monotone nondecreasing with respect to set inclusion; i.e., if $K_{i}, L_{i}$ $\in \mathscr{K}^{n}$, and $K_{i} \subset L_{i}$, then

$$
V\left(K_{1}, \ldots, K_{n}\right) \leq V\left(L_{1}, \ldots, L_{n}\right) .
$$

6 . If $K_{1}, \ldots, K_{i} \in \mathscr{K}^{n}$, and $\mathrm{C}=\left(K_{1}, \ldots, K_{i}\right)$, then the functional $V_{i}(\cdot, \mathbf{C})$ is a valuation; i.e., for $K, L \in \mathscr{K}^{n}$, such that $K \cup L \in \mathscr{K}^{n}$,

$$
V_{i}(K \cup L, \mathbf{C})+V_{i}(K \cap L, \mathbf{C})=V_{i}(K, \mathbf{C})+V_{i}(L, \mathbf{C}) .
$$

Properties $1_{\mathrm{v}}$ and $2 \mathrm{v}$ uniquely determine the mixed volume functional (as can be seen from its definition (0.4)).

One of the fundamental inequalities for mixed volumes is the Minkowski (mixed volume) inequality: If $K, L \in \mathscr{K}_{0}^{n}$, then

$$
V_{1}(K, L)^{n} \geq V(K)^{n-1} V(L),
$$

with equality if and only if $K$ and $L$ are homothetic. In fact a general version of Minkowski's inequality holds: If $K, L \in \mathscr{K}_{0}^{n}$, and $0 \leq i<n-1$, then

$$
W_{i}(K, L)^{n-i} \geq W_{i}(K)^{n-i-1} W_{i}(L),
$$

with equality if and only if $K$ and $L$ are homothetic. Of course, (1.1) is the special case $i=0$ of (1.2).

An obvious immediate consequence of the Minkowski inequality is that if $K, L \in \mathscr{M} \subset \mathscr{K}_{0}^{n}$, and if either

$$
V_{1}(K, Q)=V_{1}(L, Q) \quad \text { for all } Q \in \mathscr{M}
$$

or

$$
V_{1}(Q, K)=V_{1}(Q, L) \text { for all } Q \in \mathscr{M}
$$

hold, then it follows that $K=L$, up to translation.

An important generalization of the Minkowski inequality is the AleksandrovFenchel inequality: If $K_{1}, \ldots, K_{n} \in \mathscr{K}_{0}^{n}, 1 \leq m \leq n$, and $\mathbf{C}=\left(K_{m+1}, \ldots, K_{n}\right)$, then

$$
V\left(K_{1}, \ldots, K_{n}\right)^{m} \geq \prod_{j=1}^{m} V_{n-m}\left(K_{j}, \mathbf{C}\right) .
$$

Unfortunately, the equality conditions of this inequality are, in general, unknown (see the discussion in Schneider [34]). 
Let $K_{1}, \ldots, K_{n} \in \mathscr{K}_{0}^{n}$. The special case $m=n-1$, of inequality (1.4), is

$$
V\left(K_{1}, \ldots, K_{n}\right)^{n-1} \geq V_{1}\left(K_{1}, K_{n}\right) \cdots V_{1}\left(K_{n-1}, K_{n}\right) .
$$

When inequality (1.5) is combined with the Minkowski inequality (1.1), the result is

$$
V\left(K_{1}, \ldots, K_{n}\right) \geq V\left(K_{1}\right) \cdots V\left(K_{n}\right),
$$

with equality if and only if the $K_{i}$ are homothetic.

An important special case of inequality (1.4), where the equality conditions are known, is the classical inequality between the Quermassintegrals: If $K \in$ $\mathscr{K}_{0}^{n}$, and $0 \leq i<j<n$, then

$$
\omega_{n}^{j-i} W_{i}(K)^{n-j} \leq W_{j}(K)^{n-i},
$$

with equality if and only if $K$ is a ball. Of course, the special case where $i=0$ and $j=1$ is the classical inequality between the volume and surface area of a convex body.

A simple consequence of the Minkowski inequality (and the multilinearity of mixed volumes) is the Brunn-Minkowski inequality: If $K, L \in \mathscr{K}_{0}^{n}$, then

$$
V(K+L)^{1 / n} \geq V(K)^{1 / n}+V(L)^{1 / n},
$$

with equality if and only if $K$ and $L$ are homothetic. This is the special case $i=0$ of the general inequality: If $K, L \in \mathscr{K}_{0}^{n}$, and $0 \leq i<n-1$, then

$$
W_{i}(K+L)^{1 /(n-i)} \geq W_{i}(K)^{1 /(n-i)}+W_{i}(L)^{1 /(n-i)},
$$

with equality if and only if $K$ and $L$ are homothetic. For $i=n-1$, the quantities on both sides of inequality (1.9) are equal.

A generalization of inequality (1.9) is also known (see [2, pp. 1218-1219]): If $0 \leq i<n-1, K, L, K_{1}, \ldots, K_{i} \in \mathscr{K}^{n}$, and $\mathbf{C}=\left(K_{1}, \ldots, K_{i}\right)$, then

$$
V_{i}(K+L, \mathrm{C})^{1 /(n-i)} \geq V_{i}(K, \mathrm{C})^{1 /(n-i)}+V_{i}(L, \mathbf{C})^{1 /(n-i)} .
$$

The equality conditions for inequality (1.10) are not yet known.

\section{PRojection AND MiXed PROJection bodies}

The projection body, $\Pi K$, of the body $K \in \mathscr{K}^{n}$ is defined as the convex figure whose support function is given, for $u \in S^{n-1}$, by

$$
h(\Pi K, u)=v\left(K^{u}\right) .
$$

It follows from $(0.5)$ that

$$
h(\Pi K, u)=n V_{1}(K, \bar{u}) .
$$

Since for $u^{\prime} \in S^{n-1}, h\left(\bar{u}, u^{\prime}\right)=\left|u \cdot u^{\prime}\right| / 2$, it follows from (0.6) that

$$
h(\Pi K, u)=\frac{1}{2} \int_{S^{n-1}}\left|u \cdot u^{\prime}\right| d S\left(K, u^{\prime}\right) .
$$

From (2.1c), one easily sees that the homogeneous extension of degree 1 (to $\left.\mathbf{R}^{n}\right)$ of $h(\Pi K, \cdot)$ is a convex function and hence $\Pi K$ is a convex figure.

It is easy to see, from (2.1a), that a projection body is always centered (symmetric about the origin), and if $K$ has interior points then $\Pi K$ will have interior points as well. 
In the same manner that the ordinary volume functional, and Minkowski addition, leads to mixed volumes, the projection operator, and Minkowski addition, leads to mixed projection bodies: If $K_{1}, \ldots, K_{r} \in \mathscr{K}^{n}$ and $\lambda_{1}, \ldots, \lambda_{r} \geq$ 0 , then the projection body of the Minkowski linear combination

$$
\lambda_{1} K_{1}+\cdots+\lambda_{r} K_{r} \in \mathscr{K}^{n},
$$

can be written as a symmetric homogeneous polynomial of degree $n-1$ in the $\lambda_{i}$ :

$$
\Pi\left(\lambda_{1} K_{1}+\cdots+\lambda_{r} K_{r}\right)=\sum \lambda_{i_{1}} \cdots \lambda_{i_{n-1}} \Pi_{i_{1} \cdots i_{n-1}},
$$

where the sum is a Minkowski sum (of bodies) taken over all $(n-1)$-tuples $\left(i_{1}, \ldots, i_{n-1}\right)$ of positive integers not exceeding $r$. The body $\Pi_{i_{1} \cdots i_{n-1}}$ (which by definition is required to be symmetric in its subscripts) depends only on the bodies $K_{i_{1}}, \ldots, K_{i_{n-1}}$, and is uniquely determined by (2.2); it is called the mixed projection body of $K_{i_{1}}, \ldots, K_{i_{n-1}}$, and is written as $\Pi\left(K_{i_{1}}, \ldots, K_{i_{n-1}}\right)$. If $K_{1}, \ldots, K_{n-1} \in \mathscr{K}^{n}$, and $\mathbf{C}=\left(K_{1}, \ldots, K_{i}\right)$, then $\Pi\left(\mathbf{C}, K_{i+1}, \ldots, K_{n-1}\right)$ will sometimes be used in place of $\Pi\left(K_{1}, \ldots, K_{n-1}\right)$. If $K_{i+1}=\cdots=K_{n}=K$, then write $\Pi_{i}(K, \mathrm{C})$ for $\Pi\left(K_{1}, \ldots, K_{n-1}\right)$. In particular, for $K, L \in \mathscr{K}^{n}$, write $\Pi_{i}(K, L)$ for the mixed projection body $\Pi(K, \ldots, K, L, \ldots, L)$, with $i$ copies of $L$, and $n-i-1$ copies of $K$. For the projection body $\Pi_{i}(K, B)$ simply write $\Pi_{i} K$. (Note that this indexing follows [18, 19, 30], and differs from that used in the survey of Schneider and Weil [35].)

Observe that the projection of a Minkowski linear combination of figures is equal to the corresponding Minkowski linear combination of the projections of the figures. From this observation, one easily deduces (2.2) from (2.1a), together with (0.3) and (0.4). In fact from this follows that

$$
\left.h\left(\Pi\left(K_{1}, \ldots, K_{n-1}\right), u\right)\right)=v\left(K_{1}^{u}, \ldots, K_{n-1}^{u}\right) .
$$

Thus, from (0.5), it follows that

$$
\left.h\left(\Pi\left(K_{1}, \ldots, K_{n-1}\right), u\right)\right)=n V\left(K_{1}, \ldots, K_{n-1}, \bar{u}\right),
$$

and from (0.6), it follows that

$$
h\left(\Pi\left(K_{1}, \ldots, K_{n-1}\right), u\right)=\frac{1}{2} \int_{S^{n-1}}\left|u \cdot u^{\prime}\right| d S\left(K_{1}, \ldots, K_{n-1} ; u^{\prime}\right) .
$$

The uniqueness of the coefficients (bodies in $\mathscr{K}^{n}$ ) in (2.2) is easily observed to follow from the corresponding property of mixed volumes if $(2.2)$ is rewritten in terms of support functions, and (0.3) is used.

Just as the (ordinary) volume functional, and Minkowski combinations, produces the polylinearized form known as mixed volumes, the projection operator,

$$
\Pi: \mathscr{K}^{n} \longrightarrow \mathscr{K}^{n} \text {, }
$$

which takes $K$ into $\Pi K$, produces the mixed projection operator

$$
\Pi: \underbrace{\mathscr{R}^{n} \times \cdots \times \mathscr{K}^{n}}_{n-1} \longrightarrow \mathscr{K}^{n}
$$

The following is a list of the basic properties of the mixed projection operator. From (2.3b), together with (0.3) and $1_{v}$, it follows that: 
$1_{p}$. The projection operator is symmetric and multilinear with respect to Minkowski linear combinations; i.e., if $K, K^{\prime}, K_{2}, \ldots, K_{n-1} \in \mathscr{K}^{n}, \lambda, \lambda^{\prime} \geq 0$, and $\mathbf{C}=\left(K_{2}, \ldots, K_{n-1}\right)$, then

$$
\Pi\left(\lambda K+\lambda^{\prime} K^{\prime}, \mathbf{C}\right)=\lambda \Pi(K, \mathbf{C})+\lambda^{\prime} \Pi\left(K^{\prime}, \mathbf{C}\right) .
$$

From (2.3a), (2.1a), and $2 \mathrm{v}$, it follows that:

$2 p$. The diagonal form of the mixed projection operator reduces to the ordinary projection operator; i.e., for $K \in \mathscr{K}^{n}$,

$$
\Pi(K, \ldots, K)=\Pi K .
$$

From $(2.3 b)$ and $3 v$, it can be seen that:

3 . The mixed projection operator is continuous with respect to the Hausdorff metric.

It was shown by Petty [25] (alternate proofs can be found in [7] and [22]) that for $K \in \mathscr{K}^{n}$ and $\phi \in S L(n)$,

$$
\Pi \phi K=\phi^{-t} \Pi K,
$$

where $\phi^{-t}$ is the transpose of the inverse of $\phi$. Suppose, $K_{1}, \ldots, K_{r} \in \mathscr{K}^{n}$, $\lambda_{1}, \ldots, \lambda_{r} \geq 0$, and $\phi \in S L(n)$. Obviously, from the definition of a Minkowski linear combination, it follows that

$$
\phi\left(\lambda_{1} K_{1}+\cdots+\lambda_{r} K_{r}\right)=\lambda_{1} \phi K_{1}+\cdots+\lambda_{r} \phi K_{r} .
$$

From this observation, together with (2.4), and the definition of mixed projection bodies (2.2), it follows immediately that:

$4_{p}$. If $K_{1}, \ldots, K_{n-1} \in \mathscr{K}^{n}$, and $\phi \in S L(n)$, then

$$
\Pi\left(\phi K_{1}, \ldots, \phi K_{n-1}\right)=\phi^{-t} \Pi\left(K_{1}, \ldots, K_{n-1}\right),
$$

and since $\left|\operatorname{det}\left(\phi^{-t}\right)\right|=1$, that

$$
V\left(\Pi\left(\phi K_{1}, \ldots, \phi K_{n-1}\right)\right)=V\left(\Pi\left(K_{1}, \ldots, K_{n-1}\right)\right) .
$$

From $(2.3 b)$ and $5_{v}$, it can be seen that:

$5 \mathrm{p}$. The mixed projection operator is monotone nondecreasing with respect to set inclusion; i.e., if $K_{i}, L_{i} \in \mathscr{K}^{n}$, and $K_{i} \subset L_{i}$, then

$$
\Pi\left(K_{1}, \ldots, K_{n-1}\right) \subset \Pi\left(L_{1}, \ldots, L_{n-1}\right) .
$$

From $6_{\mathrm{v}}$ and $(2.3 \mathrm{~b})$, one has:

6 . If $K_{1}, \ldots, K_{i} \in \mathscr{K}^{n}$, and $\mathbf{C}=\left(K_{1}, \ldots, K_{i}\right)$, then the functional $\Pi_{i}\left(\cdot\right.$, C) is a valuation; i.e., for $K, L \in \mathscr{K}^{n}$, such that $K \cup L \in \mathscr{K}^{n}$,

$$
\Pi_{i}(K \cup L, \mathbf{C})+\Pi_{i}(K \cap L, \mathbf{C})=\Pi_{i}(K, \mathbf{C})+\Pi_{i}(L, \mathbf{C}) .
$$

Property $6_{p}$ is also a consequence of $(2.3 \mathrm{c})$, when combined with the observation of Schneider [29] regarding the valuation property of the surface area measures.

Note that properties $1_{p}$ and $2 p$ characterize the mixed projection operator.

It is well known, and not difficult to show, that the projection body $\Pi K$, of a polytope $K \in \mathscr{K}^{n}$, is a polytope. Also if $L_{1}, \ldots, L_{r} \in \mathscr{K}^{n}$ and $\lambda_{1}, \ldots, \lambda_{r}>0$, then it is easily shown (see, for example, Grünbaum [15]) that the Minkowski linear combination, $\lambda_{1} L_{1}+\cdots+\lambda_{r} L_{r}$, is a polytope if each $L_{i}$ is a polytope. From these facts, together with definition (2.2), follows: 
$7_{\mathbf{p}}$. If $K_{1}, \ldots, K_{n-1} \in \mathscr{K}^{n}$, are polytopes then $\Pi\left(K_{1}, \ldots, K_{n-1}\right)$ is a polytope.

\section{AN IDENTITY FOR MIXED PROJECTION BODIES}

An easy identity for mixed projection bodies, which involves mixed volumes, will facilitate a number of proofs given later.

Lemma 3.1. If $K_{1}, \ldots, K_{n-1}, L_{1}, \ldots, L_{n-1} \in \mathscr{K}^{n}$, then

$$
\begin{aligned}
& V\left(K_{1}, \ldots, K_{n-1}, \Pi\left(L_{1}, \ldots, L_{n-1}\right)\right) \\
& \quad=V\left(L_{1}, \ldots, L_{n-1}, \Pi\left(K_{1}, \ldots, K_{n-1}\right)\right) .
\end{aligned}
$$

Proof. If the convex figures involved all have interior points, then from $(0.6)$ and $(2.3 \mathrm{c})$ it follows that

$$
\begin{aligned}
2 n V\left(K_{1}, \ldots, K_{n-1}, \Pi\left(L_{1}, \ldots, L_{n-1}\right)\right) & \\
& =\int_{S^{n-1}} \int_{S^{n-1}}\left|u \cdot u^{\prime}\right| d S\left(L_{1}, \ldots, L_{n-1} ; u^{\prime}\right) d S\left(K_{1}, \ldots, K_{n-1} ; u\right) .
\end{aligned}
$$

Change the order of integration, and again use (0.6) and (2.3) to get (3.1). For arbitrary figures in $\mathscr{K}^{n}$, a standard limit argument will now yield the identity of the lemma.

Some special cases of (3.1) will frequently be used: For $K_{1}=\cdots=K_{n-i-1}=$ $K$, and $K_{n-i}=\cdots=K_{n-1}=B$, Lemma 3.1 reduces to

Lemma 3.2. If $K, L_{1}, \ldots, L_{n-1} \in \mathscr{K}^{n}$ and $0 \leq i<n-1$, then

$$
W_{i}\left(K, \Pi\left(L_{1}, \ldots, L_{n-1}\right)\right)=V\left(L_{1}, \ldots, L_{n-1}, \Pi_{i} K\right) \text {. }
$$

A useful special case of (3.2) is that for $K, L, M \in \mathscr{K}^{n}$, and $0 \leq i<n-1$,

$$
W_{i}\left(K, \Pi_{1}(L, M)\right)=V\left(L, \ldots, L, M, \Pi_{i} K\right) .
$$

In particular, when $L=M,(3.3)$ becomes

$$
W_{i}(K, \Pi L)=V_{1}\left(L, \Pi_{i} K\right) .
$$

If $K_{1}=\cdots=K_{n-i-1}=K$, while $K_{n-i}=\cdots=K_{n-1}=B$, and $L_{1}=\cdots=$ $L_{n-j-1}=L$, while $L_{n-j}=\cdots=L_{n-1}=B$, then Lemma 3.1 becomes

Lemma 3.3. If $K, L \in \mathscr{K}^{n}$, and $0 \leq i, j<n-1$, then

$$
W_{i}\left(K, \Pi_{j} L\right)=W_{j}\left(L, \Pi_{i} K\right) .
$$

The special case of (3.5), where $i=j=0$ is well known (see, for example, [20]).

Take $L_{1}=\cdots=L_{n-1}=B$ in Lemma 3.1 , note that $\Pi(B, \ldots, B)=\Pi B=$ $\omega_{n-1} B$, use $1_{v}$, and get

Lemma 3.4. If $K_{1}, \ldots, K_{n-1} \in \mathscr{K}^{n}$, then

$$
W_{n-1}\left(\Pi\left(K_{1}, \ldots, K_{n-1}\right)\right)=\omega_{n-1} V\left(K_{1}, \ldots, K_{n-1}, B\right) .
$$

For $K_{1}=\cdots=K_{n-2}=K$, and $K_{n-1}=L$, identity (3.6) becomes

$$
W_{n-1}\left(\Pi_{1}(K, L)\right)=\omega_{n-1} W_{1}(K, L) .
$$


The special case of (3.6), where $K_{1}=\cdots=K_{n-i-1}=K$, and $K_{n-i}=\cdots=$ $K_{n-1}=B$, can be rewritten as

$$
W_{n-1}\left(\Pi_{i} K\right)=\omega_{n-1} W_{i+1}(K) .
$$

In order to make the proofs that follow less cluttered, the following shorthand notation will be used: If $k$ is an integer, then $k^{\prime}$ and $k^{\prime \prime}$ will be used in place of $n-k$ and $n-k-1$, respectively.

\section{The MINKOWSKI INEQUALITY FOR MIXED PROJECTION BODIES}

The following Minkowski inequality for mixed projection bodies will be established: For $K, L \in \mathscr{K}_{0}^{n}$,

$$
V\left(\Pi_{1}(K, L)\right)^{n-1} \geq V(\Pi K)^{n-2} V(\Pi L),
$$

with equality if and only if $K$ and $L$ are homothetic. This is the special case $i=0$ of:

Theorem 4.1. If $K, L \in \mathscr{K}_{0}^{n}$, and $0 \leq i<n$, then

$$
W_{i}\left(\Pi_{1}(K, L)\right)^{n-1} \geq W_{i}(\Pi K)^{n-2} W_{i}(\Pi L),
$$

with equality if and only if $K$ and $L$ are homothetic.

Proof. First the case where $i<n-1$. Suppose $Q \in \mathscr{K}_{0}^{n}$. From (3.1), inequality (1.5), and (3.2),

$$
\begin{aligned}
W_{i}\left(Q, \Pi_{1}(K, L)\right)^{n-1} & =V\left(K, \ldots, K, L, \Pi_{i} Q\right)^{n-1} \\
& \geq V_{1}\left(K, \Pi_{i} Q\right)^{n-2} V_{1}\left(L, \Pi_{i} Q\right) \\
& =W_{i}(Q, \Pi K)^{n-2} W_{i}(Q, \Pi L) .
\end{aligned}
$$

Now apply the general Minkowski inequality (1.2) twice, and get

$$
W_{i}\left(Q, \Pi_{1}(K, L)\right)^{n-1} \geq W_{i}(Q)^{(n-1) i^{\prime \prime} / i^{\prime}} W_{i}(\Pi K)^{(n-2) / i^{\prime}} W_{i}(\Pi L)^{1 / i^{\prime}},
$$

with equality if and only if $Q, \Pi K$, and $\Pi L$ are homothetic. In inequality $\left(4.1_{1}\right)$, take $\Pi_{1}(K, L)$ for $Q$, and since $W_{i}(Q, Q)=W_{i}(Q)$, obtain inequality (4.1).

Suppose there is equality in inequality (4.1):

$$
W_{i}\left(\Pi_{1}(K, L)\right)^{n-1}=W_{i}(\Pi K)^{n-2} W_{i}(\Pi L) .
$$

From the equality conditions of inequality $\left(4.1_{1}\right)$, and the fact that projection bodies are centered, it follows that there exist $\lambda, \mu>0$, such that

$$
\Pi_{1}(K, L)=\lambda \Pi K=\mu \Pi L \text {. }
$$

Now $\left(4.1_{3}\right)$ combined with $\left(4.1_{2}\right)$, shows that

$$
\lambda^{n-2} \mu=1 \text {. }
$$

Suppose $u \in S^{n-1}$. By $\left(4.1_{3}\right),(2.3 \mathrm{a})$, and (2.1a),

$$
v_{1}\left(K^{u}, L^{u}\right)=\lambda v\left(K^{u}\right) \text { and } v_{1}\left(K^{u}, L^{u}\right)=\mu v\left(L^{u}\right) \text {. }
$$

But, $\left(4.1_{5}\right)$ and $\left(4.1_{4}\right)$ give

$$
v_{1}\left(K^{u}, L^{u}\right)^{n-1}=v\left(K^{u}\right)^{n-2} v\left(L^{u}\right) .
$$


From (4.16), and the equality conditions of the Minkowski inequality (1.1), in the space $\xi_{u}$, it follows that $K^{u}$ and $L^{u}$ are homothetic.

A result of Süss [37] states that if the bodies $K^{u}$ and $L^{u}$ are homothetic, for all $u \in S^{n-1}$, then $K$ and $L$ are homothetic. This establishes the equality conditions for the case $i<n-1$.

The case $i=n-1$ is particularly simple. From (3.7) and (3.8), it follows that inequality (4.1), for $i=n-1$, is just a disguised version of inequality (1.2), for $i=1$.

By using the inequality of Theorem 4.1 , and other inequalities which will be established, a general version of Theorem 4.1 will be obtained in the next section.

A somewhat surprising consequence of Theorem 4.1 is the following version, for mixed projection bodies, of the uniqueness result (1.3):

Theorem 4.2. If $K, L \in \mathscr{M} \subset \mathscr{K}_{0}^{n}$, and $0 \leq i<n$, and if either

$$
W_{i}\left(\Pi_{1}(K, Q)\right)=W_{i}\left(\Pi_{1}(L, Q)\right) \text { for all } Q \in \mathscr{M}
$$

or

$$
W_{i}\left(\Pi_{1}(Q, K)\right)=W_{i}\left(\Pi_{1}(Q, L)\right) \text { for all } Q \in \mathscr{M}
$$

hold, then it follows that $K=L$, up to translation.

Proof. Suppose that (4.2a) holds. Take $K$ for $Q$ in (4.2a), use Theorem (4.1) and $2 \mathrm{p}$, and get

$$
W_{i}(\Pi K) \geq W_{i}(\Pi L),
$$

with equality if and only if $K$ and $L$ are homothetic. Take $L$ for $Q$ in (4.2a), use Theorem 4.1 and $2 \mathrm{p}$, and get $W_{i}(\Pi L) \geq W_{i}(\Pi K)$. Hence, there is equality in $\left(4.2_{1}\right)$ and thus, there exists a $\lambda>0$ for which $K$ and $\lambda L$ are translates. But equality in $\left(4.2_{1}\right)$ implies that $\lambda=1$.

Exactly the same sort of argument shows that condition (4.2b) implies that $K$ and $L$ must be translates.

\section{THE ALEKSANDROV-FeNCHEL INEQUALITY FOR MIXED PROJECTION BODIES}

The Aleksandrov-Fenchel inequality, for mixed projection bodies, which will be proven is: If $K_{1}, \ldots, K_{n-1} \in \mathscr{K}^{n}$, and $\mathbf{C}=\left(K_{m+1}, \ldots, K_{n-1}\right)$, then

$$
V\left(\Pi\left(K_{1}, \ldots, K_{n-1}\right)\right)^{m} \geq \prod_{j=1}^{m} V\left(\Pi_{n-m-1}\left(K_{j}, \mathbf{C}\right)\right) \text {. }
$$

This is the special case $i=0$ of

Theorem 5.1. If $K_{1}, \ldots, K_{n-1} \in \mathscr{K}^{n}, \mathbf{C}=\left(K_{m+1}, \ldots, K_{n-1}\right)$, and $0<m<$ $n-1$, then

$$
W_{i}\left(\Pi\left(K_{1}, \ldots, K_{n-1}\right)\right)^{m} \geq \prod_{j=1}^{m} W_{i}\left(\Pi_{n-m-1}\left(K_{j}, \mathbf{C}\right)\right) .
$$

Proof. From (3.6), it follows that for $i=n-1$, inequality (5.1) reduces to inequality (1.4). Hence, assume $i<n-1$.

From (3.2), it follows that for $Q \in \mathscr{K}^{n}$, 


$$
W_{i}\left(Q, \Pi\left(K_{1}, \ldots, K_{n-1}\right)\right)=V\left(K_{1}, \ldots, K_{n-1}, \Pi_{i} Q\right) .
$$

Let $\mathbf{D}=\left(K_{m+1}, \ldots, K_{n-1}, \Pi_{i} Q\right)$. From inequality (1.4), it follows that

$$
V\left(K_{1}, \ldots, K_{n-1}, \Pi_{i} Q\right)^{m} \geq \prod_{j=1}^{m} V_{m^{\prime}}\left(K_{j}, \mathbf{D}\right) .
$$

But

$$
V_{m^{\prime}}\left(K_{j}, \mathbf{D}\right)=V\left(K_{j}, \ldots, K_{j}, K_{m+1}, \ldots, K_{n-1}, \Pi_{i} Q\right),
$$

and hence from (3.2) we get

$$
V_{m^{\prime}}\left(K_{j}, \mathbf{D}\right)=W_{i}\left(Q, \Pi_{m^{\prime \prime}}\left(K_{j}, \mathbf{C}\right)\right) .
$$

From inequality (1.2), and $\left(5.1_{3}\right)$, it follows that

$$
V_{m^{\prime}}\left(K_{j}, \mathbf{D}\right)^{i^{\prime}} \geq W_{i}(Q)^{i^{\prime \prime}} W_{i}\left(\Pi_{m^{\prime \prime}}\left(K_{j}, \mathbf{C}\right)\right) .
$$

Combine this with $\left(5.1_{1}\right)$ and $\left(5.1_{2}\right)$ to obtain

$$
W_{i}\left(Q, \Pi\left(K_{1}, \ldots, K_{n-1}\right)\right)^{m i^{\prime}} \geq W_{i}(Q)^{m i^{\prime \prime}} \prod_{j=1}^{m} W_{i}\left(\Pi_{m^{\prime \prime}}\left(K_{j}, \mathbf{C}\right)\right) .
$$

Now take $Q=\Pi\left(K_{1}, \ldots, K_{n-1}\right)$, recall that $W_{i}(Q, Q)=W_{i}(Q)$, and from $\left(5.1_{4}\right)$ get inequality $(5.1)$.

From the case $m=n-2$ of inequality (5.1), it follows that

$$
W_{i}\left(\Pi\left(K_{1}, \ldots, K_{n-1}\right)\right)^{n-2} \geq W_{i}\left(\Pi_{1}\left(K_{1}, K_{n-1}\right)\right) \cdots W_{i}\left(\Pi_{1}\left(K_{n-2}, K_{n-1}\right)\right) .
$$

Combine inequalities (5.2) and (4.1), and the result is

Theorem 5.2. If $K_{1}, \ldots, K_{n-1} \in \mathscr{K}_{0}^{n}$, and $0 \leq i<n$, then

$$
W_{i}\left(\Pi\left(K_{1}, \ldots, K_{n-1}\right)\right)^{n-1} \geq W_{i}\left(\Pi K_{1}\right) \cdots W_{i}\left(\Pi K_{n-1}\right),
$$

with equality if and only if the $K_{i}$ are homothetic.

Theorem 5.2 is the case $m=n-1$ of Theorem 5.1, with equality conditions.

The special case of Theorem 5.2, where we have $K_{1}=\cdots=K_{n-1-j}=K$, and $K_{n-j}=\cdots=K_{n-1}=L$, provides the promised generalization of Theorem 4.1.

Theorem 5.3. If $K, L \in \mathscr{K}_{0}^{n}$, while $0 \leq i<n$, and $0<j<n-1$, then

$$
W_{i}\left(\Pi_{j}(K, L)\right)^{n-1} \geq W_{i}(\Pi K)^{n-j-1} W_{i}(\Pi L)^{j},
$$

with equality if and only if $K$ and $L$ are homothetic.

Exactly the same method of proof that yielded Theorem 4.2 from Theorem 4.1 gives

Theorem 5.4. If $K, L \in \mathscr{M} \subset \mathscr{K}_{0}^{n}$, and $0 \leq i<n$, while $0<j<n-1$, and if either 


$$
W_{i}\left(\Pi_{j}(K, Q)\right)=W_{i}\left(\Pi_{j}(L, Q)\right) \quad \text { for all } Q \in \mathscr{M}
$$

or

$$
W_{i}\left(\Pi_{j}(Q, K)\right)=W_{i}\left(\Pi_{j}(Q, L)\right) \quad \text { for all } Q \in \mathscr{M}
$$

hold, then it follows that $K=L$, up to translation.

An immediate consequence of Theorem 5.3 is that for $K \in \mathscr{K}_{0}^{n}, 0 \leq i<n$, and $0<j<n-1$,

$$
W_{i}\left(\Pi_{j} K\right)^{n-1} \geq \omega_{n-1}^{(n-i) j} \omega_{n}^{j} W_{i}(\Pi K)^{n-j-1},
$$

with equality if and only if $K$ is a ball. However, the equality conditions for a more general inequality than (5.6) can be obtained.

Theorem 5.5. If $K \in \mathscr{K}_{0}^{n}$, and $0 \leq i<j<n-1$, while $0 \leq m<n$, then

$$
W_{m}\left(\Pi_{j} K\right)^{n-i-1} \geq \omega_{n-1}^{(n-m)(j-i)} \omega_{n}^{j-i} W_{m}\left(\Pi_{i} K\right)^{n-j-1},
$$

with equality if and only if $K$ is a ball.

Proof. From (3.8), it follows that the case $m=n-1$ of inequality (5.7) reduces to (1.7), and hence, it may be assumed that $m<n-1$.

Suppose $Q \in \mathscr{K}_{0}^{n}$. From (3.5),

$$
W_{m}\left(Q, \Pi_{j} K\right)=W_{j}\left(K, \Pi_{m} Q\right) .
$$

From inequality (1.4), with $\mathbf{C}=\left(B, \ldots, B, \Pi_{m} Q\right)$, with $i$ copies of $B$, it follows that

$$
W_{j}\left(K, \Pi_{m} Q\right)^{i^{\prime \prime}} \geq W_{n-1}\left(\Pi_{m} Q\right)^{j-i} W_{i}\left(K, \Pi_{m} Q\right)^{j^{\prime \prime}} .
$$

From (3.8) and inequality (1.7), it follows that

$$
W_{n-1}\left(\Pi_{m} Q\right) \geq \omega_{n-1} \omega_{n}^{1 / m^{\prime}} W_{m}(Q)^{m^{\prime \prime} / m^{\prime}},
$$

with equality if and only if $Q$ is a ball.

For the second term on the right of $\left(5.7_{2}\right)$, note that by (3.5),

$$
W_{i}\left(K, \Pi_{m} Q\right)=W_{m}\left(Q, \Pi_{i} K\right) .
$$

Apply inequality (1.2) to the quantity on the right and get:

$$
W_{i}\left(K, \Pi_{m} Q\right)^{m^{\prime}} \geq W_{m}(Q)^{m^{\prime \prime}} W_{m}\left(\Pi_{i} K\right),
$$

with equality if and only if $Q$ and $\Pi_{i} K$ are homothetic.

Now take $Q=\Pi_{j} K$; combine $\left(5.7_{1}\right)$ with $\left(5.7_{2}\right),\left(5.7_{3}\right)$, and $\left(5.7_{4}\right)$, and the result is the promised inequality of the theorem.

Suppose there is equality in inequality (5.7):

$$
W_{m}\left(\Pi_{j} K\right)^{i^{\prime \prime}}=\omega_{n-1}^{(j-i) m^{\prime}} \omega_{n}^{j-i} W_{m}\left(\Pi_{i} K\right)^{j^{\prime \prime}} .
$$

From the equality conditions of inequalities $\left(5.7_{3}\right)$ and $\left(5.7_{4}\right)$, this implies that $\Pi_{i} K$ and $\Pi_{j} K$ must be centered balls. Thus there exist $\lambda, \mu>0$, such that

$$
\Pi_{i} K=\lambda B \text { and } \Pi_{j} K=\mu B .
$$

But $\left(5.7_{6}\right)$, together with $\left(5.7_{5}\right)$, gives

$$
\mu^{i^{\prime \prime}}=\omega_{n-1}^{j-i} \lambda^{j^{\prime \prime}} .
$$


Fix $u \in S^{n-1}$. From (2.3a) and (5.76), it follows that

$$
w_{i}\left(K^{u}\right)=\lambda \text { and } w_{j}\left(K^{u}\right)=\mu .
$$

But $\left(5.7_{8}\right)$ combined with $\left(5.7_{7}\right)$ shows that there is equality in inequality (1.7), between the $(n-1)$-dimensional Quermassintegrals of $K^{u}$, in the space $\xi_{u}$, and thus $K^{u}$ is a ball. Since all projections of $K$ are balls, it follows from the previously mentioned result of Süss, that $K$ must be a ball.

\section{The BRUNN-MINKOWSKI INEQUALITY FOR MIXED PROJECTION BODIES}

The Brunn-Minkowski inequality for projection bodies, which will be established is: If $K, L \in \mathscr{K}_{0}^{n}$, then

$$
V(\Pi(K+L))^{1 / n(n-1)} \geq V(\Pi K)^{1 / n(n-1)}+V(\Pi L)^{1 / n(n-1)},
$$

with equality if and only if $K$ and $L$ are homothetic. In fact a considerably more general inequality (with equality conditions) will be established. However, first an inequality of this type, without equality conditions, is given.

Theorem 6.1. If $0 \leq i<n$, while $0 \leq j<n-1$, and $K, L, M_{1}, \ldots, M_{i}$, $M_{1}^{\prime}, \ldots, M_{j}^{\prime} \in \mathscr{K}^{n}, \mathbf{C}=\left(M_{1}, \ldots, M_{i}\right)$, and $\mathbf{D}=\left(M_{1}^{\prime}, \ldots, M_{j}^{\prime}\right)$, then

$$
\begin{aligned}
& V_{i}\left(\Pi_{j}(K+L, \mathbf{D}), \mathbf{C}\right)^{1 /(n-i)(n-j-1)} \\
& \quad \geq V_{i}\left(\Pi_{j}(K, \mathbf{D}), \mathbf{C}\right)^{1 /(n-i)(n-j-1)}+V_{i}\left(\Pi_{j}(L, \mathbf{D}), \mathbf{C}\right)^{1 /(n-i)(n-j-1)} .
\end{aligned}
$$

Proof. If $j=n-2$, then from $1_{\mathbf{p}}$, it follows that

$$
\Pi_{j}(K+L, \mathbf{D})=\Pi_{j}(K, \mathbf{D})+\Pi_{j}(L, \mathbf{D}) .
$$

Hence, for $j=n-2$, the inequality of the theorem reduces to inequality (1.10). If $i=n-1$, then from (3.1), it follows that the inequality of the theorem reduces to (1.10). Thus, only the cases where $j<n-2$, and $i<n-1$ need be treated.

Suppose $Q \in \mathscr{K}^{n}$. From (3.1) it follows that

$$
V\left(Q, i^{\prime \prime} ; \mathbf{C} ; \Pi_{j}(K+L, \mathbf{D})\right)=V\left(K+L, j^{\prime \prime} ; \mathbf{D} ; \Pi_{i}(Q, \mathbf{C})\right) .
$$

Inequality (1.10) shows that

$$
\begin{aligned}
V(K & \left.+L, j^{\prime \prime} ; \mathbf{D} ; \Pi_{i}(Q, \mathbf{C})\right)^{1 / j^{\prime \prime}} \\
& \geq V\left(K, j^{\prime \prime} ; \mathbf{D} ; \Pi_{i}(Q, \mathbf{C})\right)^{1 / j^{\prime \prime}}+V\left(L, j^{\prime \prime} ; \mathbf{D} ; \Pi_{i}(Q, \mathbf{C})\right)^{1 / j^{\prime \prime}} .
\end{aligned}
$$

But from the identity (3.1), follows

$$
V\left(K, j^{\prime \prime} ; \mathbf{D} ; \Pi_{i}(Q, \mathbf{C})\right)^{1 / j^{\prime \prime}}=V\left(Q, i^{\prime \prime} ; \mathbf{C} ; \Pi_{j}(K, \mathbf{D})\right)^{1 / j^{\prime \prime}},
$$

and hence, inequality (1.4) gives

$$
V\left(K, j^{\prime \prime} ; \mathbf{D} ; \Pi_{i}(Q, \mathbf{C})\right)^{1 / j^{\prime \prime}} \geq V_{i}(Q, \mathbf{C})^{i^{\prime \prime} / i^{\prime} j^{\prime \prime}} V_{i}\left(\Pi_{j}(K, \mathbf{D}), \mathbf{C}\right)^{1 / i^{\prime} j^{\prime \prime}} .
$$

In exactly the same way, it can be seen that

$$
V\left(L, j^{\prime \prime} ; \mathbf{D} ; \Pi_{i}(Q, \mathbf{C})\right)^{1 / j^{\prime \prime}} \geq V_{i}(Q, \mathbf{C})^{i^{\prime \prime} / i^{\prime} j^{\prime \prime}} V_{i}\left(\Pi_{j}(L, \mathbf{D}), \mathbf{C}\right)^{1 / i^{\prime} j^{\prime \prime}}
$$

Combine $\left(6.2_{1}\right),\left(6.2_{2}\right),\left(6.2_{3}\right),\left(6.2_{4}\right)$, and the result is

$$
\begin{aligned}
& V\left(Q, i^{\prime \prime} ; \mathbf{C} ; \Pi_{j}(K+L, \mathbf{D})\right)^{1 / j^{\prime \prime}} V_{i}(Q, \mathbf{C})^{-i^{\prime \prime} / i^{\prime} j^{\prime \prime}} \\
& \quad \geq V_{i}\left(\Pi_{j}(K, \mathbf{D}), \mathbf{C}\right)^{1 / i^{\prime} j^{\prime \prime}}+V_{i}\left(\Pi_{j}(L, \mathbf{D}), \mathbf{C}\right)^{1 / i^{\prime} j^{\prime \prime}} .
\end{aligned}
$$


Take $\Pi_{j}(K+L, \mathbf{D})$ for $Q$, and note that the left side of the last inequality reduces to $V_{i}\left(\Pi_{j}(K+L, \mathbf{D}), \mathbf{C}\right)^{1 / i^{\prime} j^{\prime \prime}}$, which shows that the last inequality is the inequality of the theorem.

The most interesting case of the inequality of Theorem 6.1 is the special case where $\mathbf{D}=(B, \ldots, B)$. In this case the inequality of Theorem 6.1 reads

$$
\begin{aligned}
& V_{i}\left(\Pi_{j}(K+L), \mathbf{C}\right)^{1 /(n-i)(n-j-1)} \\
& \quad \geq V_{i}\left(\Pi_{j} K, \mathbf{C}\right)^{1 /(n-i)(n-j-1)}+V_{i}\left(\Pi_{j} L, \mathbf{C}\right)^{1 /(n-i)(n-j-1)} .
\end{aligned}
$$

For the special case where also $\mathbf{C}=(B, \ldots, B)$, the equality conditions of this inequality may be easily established.

Theorem 6.2. If $K, L \in \mathscr{K}_{0}^{n}$, and $0 \leq i<n$, while $0 \leq j<n-2$, then

$$
W_{i}\left(\Pi_{j}(K+L)\right)^{1 /(n-i)(n-j-1)} \geq W_{i}\left(\Pi_{j} K\right)^{1 /(n-i)(n-j-1)}+W_{i}\left(\Pi_{j} L\right)^{1 /(n-i)(n-j-1)},
$$

with equality if and only if $K$ and $L$ are homothetic.

Proof. If $Q \in \mathscr{K}_{0}^{n}$, then (3.5) shows that

$$
W_{i}\left(Q, \Pi_{j}(K+L)\right)=W_{j}\left(K+L, \Pi_{i} Q\right) .
$$

From inequality (1.10), it follows that

$$
W_{j}\left(K+L, \Pi_{i} Q\right)^{1 / j^{\prime \prime}} \geq W_{j}\left(K, \Pi_{i} Q\right)^{1 / j^{\prime \prime}}+W_{j}\left(L, \Pi_{i} Q\right)^{1 / j^{\prime \prime}} .
$$

But from (3.5), one has

$$
W_{j}\left(K, \Pi_{i} Q\right)^{1 / j^{\prime \prime}}=W_{i}\left(Q, \Pi_{j} K\right)^{1 / j^{\prime \prime}},
$$

and hence, inequality $(1.2)$ gives

$$
W_{j}\left(K, \Pi_{i} Q\right)^{1 / j^{\prime \prime}} \geq W_{i}(Q)^{i^{\prime \prime} / i^{\prime} j^{\prime \prime}} W_{i}\left(\Pi_{j} K\right)^{1 / i^{\prime} j^{\prime \prime}},
$$

with equality if and only if $Q$ and $\Pi_{j} K$ are homothetic. In exactly the same way, one obtains

$$
W_{j}\left(L, \Pi_{i} Q\right)^{1 / j^{\prime \prime}} \geq W_{i}(Q)^{i^{\prime \prime} / i^{\prime} j^{\prime \prime}} W_{i}\left(\Pi_{j} L\right)^{1 / i^{\prime} j^{\prime \prime}},
$$

with equality if and only if $Q$ and $\Pi_{j} L$ are homothetic.

Combine $\left(6.3_{1}\right),\left(6.3_{2}\right),\left(6.3_{3}\right)$, and $\left(6.3_{4}\right)$, and the result is

(6.35) $W_{i}\left(Q, \Pi_{j}(K+L)\right)^{1 / j^{\prime \prime}} W_{i}(Q)^{-i^{\prime \prime} / i^{\prime} j^{\prime \prime}} \geq W_{i}\left(\Pi_{j} K\right)^{1 / i^{\prime} j^{\prime \prime}}+W_{i}\left(\Pi_{j} L\right)^{1 / i^{\prime} j^{\prime \prime}}$,

with equality if and only if $\Pi_{j} K, \Pi_{j} L$, and $Q$ are homothetic. Take $\Pi_{j}(K+L)$ for $Q$, and inequality $\left(6.3_{5}\right)$ becomes the inequality of Theorem 6.2 .

Suppose there is equality in the inequality of the theorem:

$$
\begin{aligned}
& W_{i}\left(\Pi_{j}(K+L)\right)^{1 /(n-i)(n-j-1)} \\
& \quad=W_{i}\left(\Pi_{j} K\right)^{1 /(n-i)(n-j-1)}+W_{i}\left(\Pi_{j} L\right)^{1 /(n-i)(n-j-1)} .
\end{aligned}
$$

From the equality conditions for inequality $\left(6.3_{5}\right)$, conclude that $\Pi_{j} K, \Pi_{j} L$, and $\Pi_{j}(K+L)$ are homothetic. Since projection bodies are centered, there exist $\lambda, \mu>0$, such that

$$
\Pi_{j} K=\lambda \Pi_{j}(K+L), \quad \text { and } \quad \Pi_{j} L=\mu \Pi_{j}(K+L) .
$$


But $\left(6.3_{7}\right)$ combined with $\left(6.3_{6}\right)$, gives

$$
1=\lambda^{1 / j^{\prime \prime}}+\mu^{1 / j^{\prime \prime}} \text {. }
$$

Suppose $u \in S^{n-1}$. Since $(K+L)^{u}=K^{u}+L^{u}$, it follows from (2.3a) and (6.37) that

$$
w_{j}\left(K^{u}\right)=\lambda w_{j}\left(K^{u}+L^{u}\right) \text { and } w_{j}\left(L^{u}\right)=\mu w_{j}\left(K^{u}+L^{u}\right) .
$$

However (6.39) combined with $(6.28)$, yields

$$
w_{j}\left(K^{u}+L^{u}\right)^{1 / j^{\prime \prime}}=w_{j}\left(K^{u}\right)^{1 / j^{\prime \prime}}+w_{j}\left(L^{u}\right)^{1 / j^{\prime \prime}} .
$$

But the equality conditions of inequality (1.9), in the space $\xi_{u}$, show that this implies that $K^{u}$ and $L^{u}$ must be homothetic. Since this holds for all $u \in S^{n-1}$, it follows, from the previously mentioned result of Süss, that $K$ and $L$ are homothetic.

The more interesting cases of Theorem 6.2 are the cases where $i=0$ or $j=0$. For the case $i=0$, Theorem 6.2 is: If $K, L \in \mathscr{K}_{0}^{n}$, and $0 \leq j<n-2$, then

$$
V\left(\Pi_{j}(K+L)\right)^{1 / n(n-j-1)} \geq V\left(\Pi_{j} K\right)^{1 / n(n-j-1)}+V\left(\Pi_{j} L\right)^{1 / n(n-j-1)},
$$

with equality if and only if $K$ and $L$ are homothetic. For the case $j=0$, Theorem 6.2 is: If $K, L \in \mathscr{K}_{0}^{n}$ and $0 \leq i<n$, then

$$
W_{i}(\Pi(K+L))^{1 /(n-i)(n-1)} \geq W_{i}(\Pi K)^{1 /(n-i)(n-1)}+W_{i}(\Pi L)^{1 /(n-i)(n-1)},
$$

with equality if and only if $K$ and $L$ are homothetic. If $i=n-1$, then by (3.8), (6.5) reduces to the special case $i=1$ and $\mathbf{C}=B$, of inequality (1.10). Of course, the promised Brunn-Minkowski inequality for projection bodies (6.1) is the special case $j=0$ of (6.4), and $i=0$ of (6.5).

\section{REFERENCES}

1. A. D. Aleksandrov, On the theory of mixed volumes of convex bodies. I: Extension of certain concepts in the theory of convex bodies, Mat. Sb. 2 (1937), 947-972. (Russian)

2. __ On the theory of mixed volumes of convex bodies. II: New inequalities between mixed volumes and their applications, Mat. Sb. 2 (1937), 1205-1238. (Russian)

3. R. Alexander, Zonoid theory and Hilbert's fourth problem, Geom. Dedicata 28 (1988), 199-211.

4. U. Betke and P. McMullen, Estimating the sizes of convex bodies from projections, J. London Math. Soc. (2) 27 (1983), 525-538.

5. E. D. Bolker, A class of convex bodies, Trans. Amer. Math. Soc. 145 (1969), 323-345.

6. T. Bonnesen and W. Fenchel, Theorie der konvexen Körper, Springer, Berlin, 1934.

7. J. Bourgain and J. Lindenstrauss, Projection bodies, Geometric Aspects of Functional Analysis (J. Lindenstrauss and V. D. Milman, eds.), Lecture Notes in Math., vol. 1317, SpringerVerlag, Berlin and New York, 1988, pp. 250-270.

8. H. Busemann, Convex surfaces, Interscience, New York, 1958.

9. G. D. Chakerian, Sets of constant relative width and constant relative brightness, Trans. Amer. Math. Soc. 129 (1967), 26-37.

10. W. Fenchel and B. Jessen, Mengenfunktionen und konvexe Körper, Danske Vid. Selskab. Mat.-fys. Medd. 16 (1938), 1-31.

11. P. R. Goodey, Instability of projection bodies, Geom. Dedicata 20 (1986), 295-305. 
12. P. R. Goodey and H. Groemer, Stability results for first order projection bodies, Proc. Amer. Math. Soc. 109 (1990), 1103-1114.

13. Y. Gordon, M. Meyer, and S. Reisner, Zonoids with minimal volume product-a new proof, Proc. Amer. Math. Soc. 104 (1988), 273-276.

14. H. Groemer, On multiple space subdivisions by zonotopes, Monatsh. Math. 86 (1978), 185-188.

15. B. Grünbaum, Convex polytopes, Interscience, New York, 1967.

16. W. Hildenbrand, Short-run production functions based on microdata, Econometrica 49 (1981), 1095-1125.

17. K. Leichtweiß, Konvexe Mengen, Springer, Berlin, 1980.

18. E. Lutwak, Mixed projection inequalities, Trans. Amer. Math. Soc. 287 (1985), 91-106.

19. _ _ Volume of mixed bodies, Trans. Amer. Math. Soc. 294 (1986), 487-500.

20. __, On some affine isoperimetric inequalities, J. Differential Geom. 23 (1986), 1-13.

21. __ Intersection bodies and dual mixed volumes, Adv. Math. 71 (1988), 232-261.

22. __ Centroid bodies and dual mixed volumes, Proc. London Math. Soc. 60 (1990), 365-391.

23. P. McMullen, On zonotopes, Trans. Amer. Math. Soc. 159 (1971), 91-109.

24. P. McMullen and R. Schneider, Valuations on convex bodies, Convexity and Its Applications (P. M. Gruber and J. M. Wills, eds.), Birkhäuser, Basel, 1983, pp. 170-247.

25. C. M. Petty, Projection bodies, Proc. Coll. Convexity, Copenhagen, 1965, Københavns Univ. Mat. Inst., 1967, pp. 234-241.

26. __ Isoperimetric problems, Proc. Conf. Convexity and Combinatorial Geometry (Univ. Oklahoma, 1971), University of Oklahoma, 1972, pp. 26-41.

27. __, Geominimal surface area, Geom. Dedicata 3 (1974), 77-97.

28. S. Reisner, Zonoids with minimal volume-product, Math. Z. 192 (1986), 339-346.

29. R. Schneider, Kinematische Berührmaße für konvexe Körper, Abh. Math. Sem. Univ. Hamburg 44 (1975), 12-23.

30. __ Rekonstruktion eines konvexen Körpers aus seinen Projektionen, Math. Nachr. 79 (1977), 325-329.

31. B Boundary structure and curvature of convex bodies, Contributions to Geometry (J. Tölke and J. M. Wills, eds.), Birkhäuser, Basel, 1979, pp. 13-59.

32. _ـ Random hyperplanes meeting a convex body, Z. Wahrsch. Verw. Gebiete 61 (1982), 379-387.

33. _ Random polytopes generated by anisotropic hyperplanes, Bull. London Math. Soc. 14 (1982), 549-553.

34. $\_$, On the Aleksandrov-Fenchel inequality, Ann. New York Acad. Sci. 440 (1985), 132-141.

35. R. Schneider and W. Weil, Zonoids and related topics, Convexity and Its Applications (P. M. Gruber and J. M. Wills, eds.), Birkhäuser, Basel, 1983, pp. 296-317.

36. R. P. Stanley, Two combinatorial applications of the Aleksandrov-Fenchel inequalities, J. Combin. Theory Ser. A 31 (1981), 56-65.

37. W. Süss, Zusammensetzung von Eikörpern und homothetische Eiflächen, Tôhoku Math. J. 35 (1932), 47-50.

38. C. Thomas, Extremum properties of the intersection densities of stationary Poisson hyperplane processes, Math. Oper. Statist. (Ser. Statist.) 15 (1984), 443-449.

39. R. A. Vitale, Expected absolute random determinants and zonoids, Ann. Appl. Probab. 1 (1991), 293-300.

40. W. Weil, Kontinuierliche Linearkombination von Strecken, Math. Z. 148 (1976), 71-84.

41. __ Zonoide und verwandte Klassen konvexer Körper, Monatsh. Math. 94 (1982), 73-84. 